\title{
Neuronal function and dysfunction of CYFIP2: from actin dynamics to early infantile epileptic encephalopathy
}

\author{
Yinhua Zhang ${ }^{1,2}$, Yeunkum Lee ${ }^{1,2}$ E Kihoon Han ${ }^{1,2, *}$ \\ Departments of ${ }^{1}$ Neuroscience, ${ }^{2}$ Biomedical Sciences, College of Medicine, Korea University, Seoul 02841, Korea
}

\begin{abstract}
The cytoplasmic FMR1-interacting protein family (CYFIP1 and CYFIP2) are evolutionarily conserved proteins originally identified as binding partners of the fragile $X$ mental retardation protein (FMRP), a messenger RNA (mRNA)-binding protein whose loss causes the fragile $X$ syndrome. Moreover, CYFIP is a key component of the heteropentameric WAVE regulatory complex (WRC), a critical regulator of neuronal actin dynamics. Therefore, CYFIP may play key roles in regulating both mRNA translation and actin polymerization, which are critically involved in proper neuronal development and function. Nevertheless, compared to CYFIP1, neuronal function and dysfunction of CYFIP2 remain largely unknown, possibly due to the relatively less well established association between CYFIP2 and brain disorders. Despite high amino acid sequence homology between CYFIP1 and CYFIP2, several in vitro and animal model studies have suggested that CYFIP2 has some unique neuronal functions distinct from those of CYFIP1. Furthermore, recent whole-exome sequencing studies identified de novo hot spot variants of CYFIP2 in patients with early infantile epileptic encephalopathy (EIEE), clearly implicating CYFIP2 dysfunction in neurological disorders. In this review, we highlight these recent investigations into the neuronal function and dysfunction of CYFIP2, and also discuss several key questions remaining about this intriguing neuronal protein. [BMB Reports 2019; 52(5): 304-311]
\end{abstract}

\section{INTRODUCTION}

Actin cytoskeleton dynamics are critically involved in various aspects of neuronal development and function, including early migration, dendritic and axonal outgrowth and branching, and synapse formation and plasticity (1-3). Consistently, several

*Corresponding author. Tel: +82-2-2286-1390; Fax: +82-2-953-6095; E-mail: neurohan@korea.ac.kr

https://doi.org/10.5483/BMBRep.2019.52.5.097

Received 10 December 2018

Keywords: Actin polymerization, Cytoplasmic FMR1-interacting protein 2 (CYFIP2), Early infantile epileptic encephalopathy (EIEE), Neuronal synapse, WAVE regulatory complex (WRC) mutations of genes encoding key regulators of the neuronal actin cytoskeleton have been associated with numerous neurodevelopmental and neuropsychiatric disorders, such as autism spectrum disorders (ASDs), intellectual disability (ID), schizophrenia (SCZ), and bipolar disorder (4-6). Among the key regulators, the WAVE regulatory complex (WRC) is a $\sim 400 \mathrm{kDa}$ heteropentameric protein complex consisting of the Wiskott-Aldrich syndrome protein family verprolinhomologous protein (WAVE), cytoplasmic FMR1-interacting protein (CYFIP), Nck-associated protein (NAP), Abelsoninteracting protein $(\mathrm{ABI})$, and hematopoietic stem progenitor cell $300(\operatorname{HSPC} 300)(7,8)$. The WRC is basally inactive toward its downstream actin-nucleator, i.e., the actin-related protein 2/3 (Arp2/3) complex, because the activity-bearing C-terminal VCA (verprolin homology, central, and acidic regions) domain of WAVE is sequestered by an intermolecular interaction with CYFIP (8). However, diverse cellular signaling can release the VCA domain from the CYFIP, enabling it to bind to and activate the Arp2/3 complex. Specifically, inositol phospholipids and the active form of the small GTPase Rac1 (Rac1-GTP) can cooperatively recruit the WRC to the plasma membrane and induce its conformational changes (8). Considering that Rac1-GTP directly binds to the CYFIP during the activation process, CYFIP is involved in both basal inhibition and Rac1-induced activation of the WRC. Furthermore, phosphorylation of several residues of WAVE, CYFIP, and ABI can also modulate the WRC activity (9-11).

Two evolutionarily highly conserved members of the CYFIP gene family, namely CYFIP1 and CYFIP2 (also referred to as SRA-1 and PIR121, respectively) exist (12). They are $\sim 145$ $\mathrm{kDa}$ proteins, with a high homology in their amino acid sequences (88\% identity and $95 \%$ similarity), suggesting similar functions at the molecular level. Originally, the CYFIP1/2 proteins were identified as binding partners of the fragile $X$ mental retardation protein (FMRP) (12), whose loss causes the fragile $X$ syndrome (FXS), which is characterized by ID, autistic behaviors, and dysmorphic features (13). Indeed, in addition to the WRC, CYFIP1 forms a complex with both the FMRP and the eukaryotic initiation factor $4 \mathrm{E}$ (elF4E) to regulate the translational initiation of target messenger RNAs (mRNAs) (14). Notably, the brain-derived neurotrophic factor (BDNF)induced activation of Rac1 has been shown to redistribute CYFIP1 from the FMRP-elF4E complex to the WRC, thereby 
orchestrating both activity-dependent local protein synthesis and actin remodeling in neuronal synapses (15). Clinically, structural abnormalities (deletions and duplications) of the chromosomal region (15q11-q13) encompassing CYFIP1 were identified in patients with ASDs and ID (16). CYFIP1 has also been associated with SCZ (17). Furthermore, several lines of Cyfip1 mutant mice expressed abnormal synaptic morphology and function, as well as either FXS- or ASD-like behaviors (18-20).

In contrast to CYFIP1, the neuronal function and dysfunction of CYFIP2 remain largely unknown, possibly given the relatively less well established association between CYFIP2 and brain disorders thus far (16). Nevertheless, despite the high sequence homology between CYFIP1 and CYFIP2, several lines of evidence suggest that they may have distinct functions in vivo. For example, both Cyfip1 and Cyfip2 null mice display lethality at different developmental time points (i.e., at early embryonic and perinatal stages, respectively) (20-23). Furthermore, recent whole-exome sequencing (WES) studies identified de novo hot spot mutations of CYFIP2 in patients with early infantile epileptic encephalopathy (EIEE) $(24,25)$. In this review, we highlight recent in vitro, animal model, and human genetic investigations revealing the neuronal function and dysfunction of CYFIP2, and discuss the key remaining issues for a better understanding of this intriguing, yet largely uncharacterized, protein in the nervous system.

\section{IN VITRO NEURONAL STUDIES}

Through the overexpression of green fluorescent protein (GFP)-tagged CYFIP1 and CYFIP2, Pathania et al. investigated the subcellular localization of these proteins in cultured rat hippocampal neurons (26). Both CYFIP1-GFP and CYFIP2-GFP exhibited punctate clusters highly localized to the dendritic spines, which are small protrusions on the neuronal dendrites representing excitatory postsynaptic compartments in mature neurons (27). Consistently, both proteins co-localized with endogenous excitatory synaptic markers, namely the Homer protein homolog (Homer) and the vesicular glutamate transporter (VGLUT). Indeed, the excitatory synaptic localization of the CYFIP1/2 proteins was further supported by several biochemical studies. For example, the CYFIP1/2 proteins were identified as components of the postsynaptic proteome through mass-spectrometry analyses of both the purified human and rodent postsynaptic density $(28,29)$. Moreover, both proteins were recognized as key nodes of the mouse in vivo postsynaptic protein interactome (30), whereas CYFIP2 was identified as an in vivo postsynaptic scaffold SH3 and multiple ankyrin repeat domains 3 (Shank3) interactor (31). In addition to their postsynaptic localization, Pathania et al. also reported that overexpression of either CYFIP1 or CYFIP2 increased dendritic branching and outgrowth of cultured hippocampal neurons (26). In the same study, the effects of Cyfip1, but not of Cyfip2, haploinsufficiency (in Cyfip $1^{+/-}$mice) on both hippocampal dendritic spine density and morphology were explored, and an increase in immature long and thin spines compared to wild-type (WT) mice was observed.

A large-scale in vivo phospho-proteomic analysis of nine mouse tissues (32) identified two brain-specific phosphorylation sites (S582 and T1067) of CYFIP2 (11). Furthermore, Lee et al. overexpressed WT, phospho-blocking (i.e., S582A and T1067A), and phospho-mimetic (i.e., S582D and T1067E) mutants of CYFIP2 in cultured hippocampal neurons to understand functional significance of such sites (11). With regard to dendritic spine regulation, overexpression of the T1067A mutant, but not that of either WT or other mutants, decreased the presence of stubby spines compared to control neurons, suggesting a dominant-negative effect of T1067A. In addition, while overexpression of WT CYFIP2 increased neurite outgrowth, the same was not true for T1067A. Intriguingly, when mapped on the crystal structure of the WRC (31), CYFIP2 T1067 was positioned at the interaction interface between the CYFIP and the NAP, and T1067 phosphorylation was predicted to weaken such an interaction (11). Lee et al. proposed a model based on these findings. It described that CYFIP2 T1067 phosphorylation, together with additional modifications of the WRC (such as bindings of inositol phospholipids and Rac1-GTP (8)), contributed to WRC activation through the disassembling of the self-inhibitory interactions among its components (11). In contrast to T1067 phosphorylation, the functional roles of CYFIP2 S582 phosphorylation, which is known to occur on the $\mathrm{N}$-terminal outer surface of CYFIP2 in the WRC, are yet to be defined (11).

\section{ANIMAL MODEL STUDIES}

The in vivo neuronal functions of CYFIP were initially characterized in the Drosophila, which expresses only one homolog of CYFIP (dCYFIP) (16). In fact, Schenck et al. demonstrated that CYFIP is specifically expressed in the central nervous system (CNS) of Drosophila, and is localized at synaptic terminals in the neuromuscular junctions (NMJs) (33). The length of the synaptic terminal was reduced in the NMJs of dCYFIP mutant larvae, compared to WT larvae, while the synaptic boutons had immature supernumerary buds (33). Notably, the reduced synaptic terminal length of dCYFIP mutants was the opposite phenotype of that observed in the Drosophila FMR1 (dFMR1) null NMJs (34). Indeed, Abekhoukh et al. determined that the NMJs of dCYFIP;dFMR1 double homozygous mutants were similar to WT NMJs, and not to those present in either of the single mutants. This suggests a genetic interaction between $d C Y F I P$ and $d F M R 1$ associated with the regulation of NMJ synaptic structures (35). Furthermore, Zhao et al. identified further detailed structural and functional changes in dCYFIP mutant NMJs (36). Specifically, enlarged synaptic vesicles and additional 
cisternae in the synaptic boutons were found through electron microscopic analysis of $d C Y F I P$ mutants. Moreover, given that $d C Y F I P$ mutants also showed functional defects related to the release of neurotransmitters under high-frequency stimulation, Zhao et al. suggested that dCYFIP regulates synaptic endocytosis by inhibiting F-actin assembly (36).

The nevermind (nev) mutant zebrafish was isolated from a large-scale forward genetic screening to identify the genes involved in retinotectal axon pathfinding $(37,38)$. Specifically, the dorsonasal axons of retinal ganglion cells (RGCs) are missorted with ventral axons in nev mutant zebrafish, and are abnormally projected through the dorsal tectum (even though they still targeted to the ventral tectum). Pittman et al. identified the nev gene to encode zebrafish Cyfip2 (39). In fact, while the zebrafish expresses both CYFIP1 and CYFIP2 (86\% identical), its CYFIP2 is $98 \%$ identical to that in humans (12). Pittman et al. also reported a broad Cyfip2 expression in the zebrafish CNS, even though the other axonal tracts of nev mutants, including those of both the primary motor neurons and Mauthner neurons, were normal (39). Furthermore, Pittman et al. found that retinal lamination was disrupted in nev mutants (39). Additionally, Cioni and colleagues identified more detailed molecular and cellular mechanisms behind the optic tract missorting in Cyfip2 mutant zebrafish (40). By combining the molecular replacement approach and time-lapse imaging of axonal growth cones, they showed the involvement of CYFIP2 in both homotypic axonal fasciculation and heterotypic axonal repulsion. Specifically, CYFIP2 was translocated to the growth cone in response to axon-axon contact, and regulated the filopodial dynamics to induce either axonal fasciculation or repulsion. Unlike Cyfip2, CRISPR/Cas9mediated deletion of Cyfip1 caused axonal growth defects in the optic tract, suggesting that CYFIP1 and CYFIP2 are specifically involved in axonal growth and sorting, respectively (40). In addition to the visual system, the CYFIP2 function in the auditory system of the zebrafish was also characterized. Marsden et al. performed an n-ethyl-n-nitrosourea (ENU)-based forward genetic screening to identify zebrafish mutants with reduced auditory startle thresholds (i.e., enhanced startle response) and isolated the Cyfip2 mutant as one of five lines having such a phenotype (41). The zebrafish startle circuit is composed of auditory afferents (VIII), excitatory spiral fiber (SF) interneurons, and Mauthner cells. Marsden et al. showed that the activity of this circuit was enhanced in Cyfip2 mutant zebrafish, as a result of either the increased excitability of SF interneurons or the enhanced excitatory synaptic input to SF interneurons (41). Although the detailed molecular mechanism underlying such a phenomenon is yet to be defined, the FMRP was not shown to be involved in this auditory phenotype of Cyfip2 mutant, as the fmr 1 mutant zebrafish presented a normal innate startle threshold.

The initial genetic evidence supporting the neuronal functions of CYFIP2 in mice derived from quantitative trait locus (QTL) analyses aimed at identification of the genetic loci contributing to the behavioral differences between the C57BL6J and C57BL/6N mouse substrains $(21,42)$. Specifically, the C57BL/6N substrain was branched out from original C57BL/6 substrain in the 1950s, and is currently the second major C57BL/6 mouse substrain. However, whole genome sequencing identified only approximately 10,000 single nucleotide polymorphisms (SNPs) between the two substrains (43). Therefore, QTL analysis can be useful for detecting the causal genetic factors underlying certain phenotypic differences between such substrains. Kumar et al. described the $\mathrm{C} 57 \mathrm{BL} / 6 \mathrm{~N}$ substrain as having a lower behavioral response (as measured by locomotor activity) to psychostimulants (such as cocaine and methamphetamine), compared to C57BL/6J (21). Through the performance of a QTL analysis, Kumar et al. identified that a nonsynonymous SNP (G to A at the $46,036,117$ base pair of chromosome 11, $\mathrm{mm} 9$ ) producing a serine-to-phenylalanine missense mutation (S968F) in CYFIP2 was responsible for the difference in the psychostimulant responses (21). Successively, Kirkpatrick et al. used a similar QTL analysis to show that such a Cyfip2 variant was also causally associated with the enhanced binge eating-like behavior of the C57BL/6N substrain, compared to the C57BL/6J substrain (42). With regard to the molecular mechanism behind such a phenomenon, Kumar et al. reported the S968F CYFIP2 proteins to be less stable than WT CYFIP2 when overexpressed in HEK293T cells (21). However, recent structural analysis of a WRC-Rac1 complex by cryo-electron microscopy suggested that the S968F CYFIP2 could cause excessive activation of the WRC by Rac1 (44). Therefore, further molecular and cellular studies are required to understand the process by which a single CYFIP2 variant changes the behavioral phenotypes in mice.

Both Cyfip1 and Cyfip2 null mice show lethality at different developmental time points. Specifically, while the Cyfip 1 null embryos die around embryonic day 9.5 (E9.5) $(20,26)$, the Cyfip2 null $\left(C_{f y i p} 2^{-1-}\right)$ mice die perinatally $(21,22)$. Recently, Zhang et al. revealed that, although Cyfip $2^{-1-}$ mice survived until E18.5, with an expected Mendelian inheritance ratio they would all die at postnatal day 0 (P0) (23). Therefore, it is conceivable that Cyfip $2^{-1-}$ mice could die very soon after birth (21). Although the detailed cause of lethality remains unknown, Zhang et al. also reported that Cyfip $2^{-1-}$ mice were smaller than either WT or heterozygous $\left(\right.$ Cyfip $\left.^{+/-}\right)$littermates at E18.5 (23). Specifically, the crown-rump length was shorter in Cyfip2 ${ }^{-1-}$ mice, suggesting defects in their body curvature. Nevertheless, both the brain size and overall cytoarchitecture were comparable between WT, Cyfip $2^{+/-}$and Cyfip $2^{-1-}$ mice at E18.5. Subsequent to an RNA-sequencing analysis, however, Zhang et al. found the extracellular matrix (ECM)-related genes to be significantly altered in the Cyfip $2^{-/-}$ embryonic cortex, compared with the WT cortex (23). Whether the abnormal expression of ECM-related genes can affect the neuronal development and function in Cyfip $2^{-1-}$ embryos, and whether such changes can be associated with 
their early postnatal lethality remains to be investigated.

Given the early postnatal lethality of Cyfip $2^{-1-}$ mice, Cyfip2 $^{+/-}$mice were used to characterize the functions of CYFIP2 in adult brains. Han et al. hypothesized that CYFIP2 could be one of the key actin-regulatory proteins mediating the dendritic spine abnormalities observed in fragile $X$ mental retardation 1 ( $F m r 1)$-null $\left(F m r 1^{-1 y}\right)$ mice, an animal model of FXS (22). In addition to the protein-protein interaction between CYFIP2 and FMRP (12), the Cyfip2 mRNA has been ranked as the ninth FMRP target among 842 FMRP-associated brain polyribosomal mRNAs (45). Therefore, it is plausible that the basal and/or activity-induced translation of Cyfip2 mRNA can be altered in $\mathrm{Fmrl}^{-l y}$ mice, possibly contributing to the abnormal actin-rich dendritic spines found in these mice. Moreover, Han and colleagues found that, similar to $\mathrm{Fmr1}^{-1 y}$ mice, adult Cyfip2 ${ }^{+/-}$mice exhibited some FXS-like behaviors, including hyper locomotor activity, reduced acoustic startle response, and enhanced pre-pulse inhibition (22). Notably, such behavioral phenotypes were aggravated in the $F m r 1^{-l y}$;Cyfip $2^{+/-}$double mutant mice. In concordance, the dendritic spine abnormality seen in the $F m r 1^{-l y}$ cortex (i.e., the increase in immature thin spines) was aggravated in double mutant mice (22). The basal levels of both the CYFIP2 and WAVE1 proteins were normal in the hippocampus and cortex of $F m r 1^{-l y}$ mice. Han et al. showed the treatment of cultured cortical neurons with a metabotropic glutamate receptor (mGluR) agonist 3,5-dihydroxyphenylglycine (DHPG) to induce the expression of CYFIP2 proteins, which was blocked by the translational inhibitor cycloheximide (22). Notably, both the DHPG-induced expression of CYFIP2 and the DHPG-induced dendritic spine remodeling were impaired in both $\mathrm{Fmr}^{-l y}$ and Cyfip2 ${ }^{+/-}$cortical neurons. Therefore, Cyfip2 mRNA can be translated in an mGluR-dependent manner, as a target of the FMRP-associated polyribosome, and newly expressed CYFIP2 proteins may be involved in dendritic spine remodeling (22). Nevertheless, the causal relationship between such molecular and cellular changes in Cyfip $2^{+/}$ neurons and the behavioral phenotypes of Cyfip $2^{+l-}$ mice remains to be further validated.

\section{CYFIP2 MUTATIONS IN EARLY INFANTILE EPILEPTIC ENCEPHALOPATHY}

In contrast to the well-established associations between CYFIP1 and either ASDs, ID, or SCZ, CYFIP2 has not been clearly associated with any brain disorders until recently (16). Although interstitial deletions of the chromosomal region 5q33.3-q35.1 harboring CYFIP2 have been identified in individuals with developmental delay, mild to severe ID, and seizures $(46,47)$, those deletions included tens of additional genes, preventing the identification of the causal gene(s). Furthermore, reduced protein expression of CYFIP2 in postmortem brains of patients with SCZ (48) and Alzheimer's disease (49) was reported, even though its causal relationship with the disease pathophysiology was not clearly established. Notably, two recent WES studies similarly identified de novo hot spot (Arg87) variants in CYFIP2 from patients with EIEE $(24,25)$. EIEE (OMIM 308350, also referred to as the Ohtahara syndrome) is a neurological disorder characterized by the occurrence of seizures, which begin within a year of birth (50). This disorder often progresses to West syndrome, which is characterized by epileptic spasms with hypsarrhythmia on the electroencephalogram (EEG) and severe psychomotor developmental delay (51).

In the first study, Nakashima et al. identified three de novo CYFIP2 variants at the Arg87 residue (Arg87Leu, Arg87Cys, and Arg87Pro) in four unrelated patients diagnosed with West syndrome (24). All four individuals presented their first seizure event prior to the age of 6 months and reported additional common features, including hypotonia, microcephaly, developmental delay, and ID. Using the available crystal structure of the WRC (8), Nakashima and colleagues descried the Arg87 residue of CYFIP1 (the solved WRC structure contains CYFIP1, not CYFIP2) to be at an interface between CYFIP1 and WAVE1 (24). Specifically, the side chain of Arg87 formed hydrogen bonds with both the main chain of Tyr151 in WAVE1 and the side chains of Glu625 and Glu690 in CYFIP1. Importantly, the three Arg87 variants were predicted to disrupt these hydrogen bonds, thereby destabilizing the interaction between CYFIP1 and WAVE1. Indeed, all three CYFIP2 variants showed weaker interaction with the purified VCA domain of the WAVE, compared to WT CYFIP2 (24). Furthermore, transfection of CYFIP2 mutants, but not WT, induced an aberrant F-actin accumulation in cultured mouse melanoma cells (24); an expected finding, given that the mutants released the VCA domain of the WAVE, leading to activation of the downstream Arp2/3 complex. In a subsequent investigation, Peng et al. performed a WES analysis of 56 Chinese families with West syndrome and identified 112 variants in 89 genes, which included a de novo Arg87Cys variant of CYFIP2 (25). Intriguingly, an in-frame deletion variant of CYFIP1 was also described. Overall, these studies suggest that de novo hot spot (Arg87) variants of CYFIP2 can be causally associated with West syndrome, potentially through the induction of aberrant WRC activation (i.e., gain-of-function) and F-actin polymerization.

\section{PERSPECTIVES AND CONCLUSIONS}

As highlighted in the current review, although a growing amount of evidence supports distinct in vivo functions of CYFIP1 and CYFIP2 $(35,40)$, the detailed mechanisms underlying such a phenomenon are largely unknown. Distinct spatiotemporal expression patterns of CYFIP1 and CYFIP2 may contribute to this $(40,52)$. For example, while a preliminary analysis found both the Cyfip2 mRNA and protein to be enriched in the cortex, compared with other brain regions of mice, those of Cyfip1 were observed to be evenly expressed in 
different brain regions (unpublished data). This may, at least partly, explain the cortical, but not hippocampal, dendritic spine changes seen in Cyfip2 ${ }^{+/-}$mice (22). Moreover, a different expression of CYFIP1 and CYFIP2 in brain non-neuronal cells cannot be excluded. Indeed, a mouse brain cell-type-specific RNA-sequencing database (http://www. brainrnaseq.org/) (53) highly detected the presence of Cyfip1 mRNA in microglia/macrophage, whereas Cyfip2 mRNA was abundant in newly-formed oligodendrocytes and neurons. In addition to their basal expression, neuronal activity-induced expression of Cyfip1 and Cyfip2 can be differentially regulated. For example, while Cyfip2 mRNA was identified as a high ranked FMRP target (45), the same was not true for Cyfip1, indicating that only the dendritic transport, stability, and local translation of Cyfip2 mRNA can be regulated by FMRP in an activity-dependent manner $(22,54)$. In accord, Cyfip2 mRNA was detected in neuronal dendrites (55). It is also possible that, despite their high sequence homology, CYFIP1 and CYFIP2 may have distinct groups of protein interactors. For example, in the original study which identified both CYFIP1/2 as direct binding partners of FMRP (12), CYFIP2, but not CYFIP1, was also shown to interact with FMRP-related proteins (FXR1P/FXR2P). Several independent investigations, using different biochemical approaches, recently identified the protein interactors specific to both CYFIP1 and CYFIP2 (15, 21, 30, 40). A side-by-side comprehensive analysis of these protein lists may provide another important insight into understanding the diverse functions of CYFIP1 and CYFIP2 in vivo.

Detailed molecular and cellular mechanisms underlying the processes by which the Arg87 variants of CYFIP2 lead to the neurological symptoms of West syndrome remain to be further investigated (Fig. 1). Nakashima et al. proposed that an aberrant increase in WRC activity and downstream actin polymerization may change the neuronal excitatory synaptic function, thereby disrupting the excitatory/inhibitory (E/I) synaptic balance (24) (Fig. 1A), which is considered one of the key mechanisms behind epilepsy, and other neurodevelopmental and neuropsychiatric disorders (56, 57). Not only postsynaptic functions, but also excitatory (and inhibitory) presynaptic functions may be regulated by CYFIP2 (Fig. 1B), since CYFIP1 regulates presynaptic terminal size and vesicle release probability in the developing hippocampus (58). Another possibility is that the change in neuronal excitability (neuronal propensity to generate action potential firing) by the CYFIP2 variants, is caused by affecting the surface expression and/or functional properties of certain ion channels. Notably, Chen et al. indicated that the WRC can potentially interact

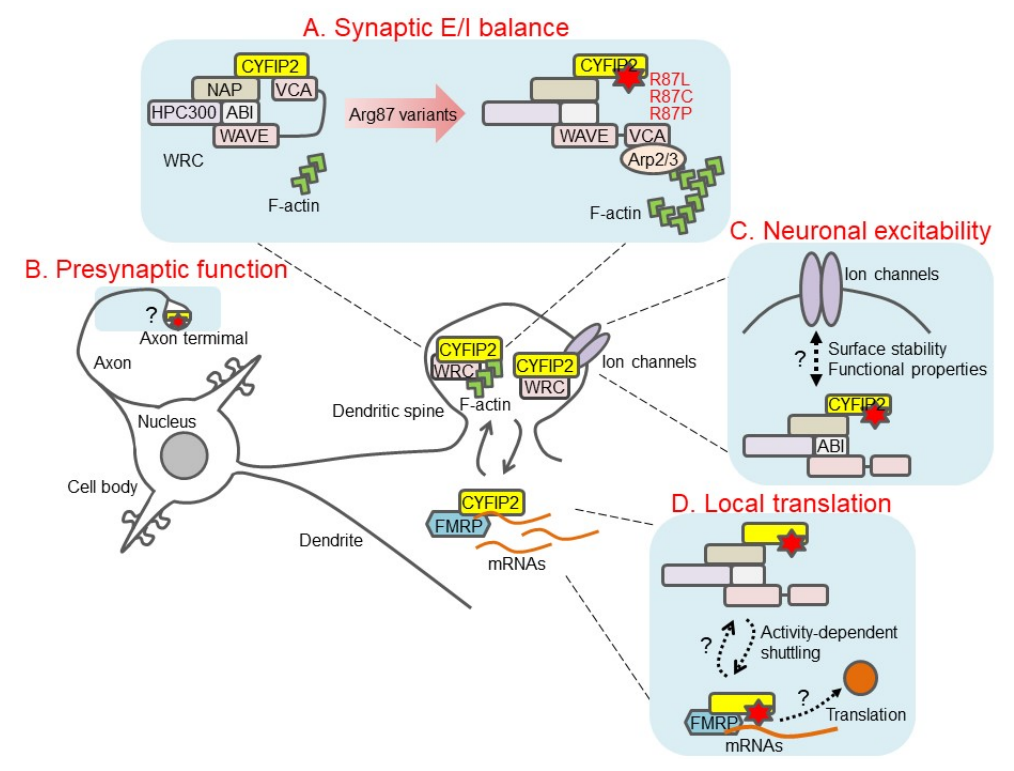

Fig. 1. Neuronal functions of CYFIP2 and potential effects of the Arg87 variants identified in the West syndrome on those functions. (A) The Arg87 variants weaken the inhibitory CYFIP2-VCA interaction, thereby leading to aberrant activation of WRC and the downstream actin polymerization. This may result in dendritic spine remodeling and change excitatory/inhibitory synaptic balance, in favor of more excitation. (B) Similar to CYFIP1, CYFIP2 may also regulate neurotransmitter release in the presynaptic compartment, and the Arg87 variants may affect this function of CYFIP2. (C) The WRC interacts with certain ion channels potentially involved in regulating neuronal excitability. Given that CYFIP and ABI of the WRC form an interaction surface toward those membrane proteins, the Arg87 variants may indirectly affect such interactions, thereby changing surface stability or other functional properties of ion channels. (D) In addition to the WRC, CYFIP2 also interacts with some mRNA-binding proteins, such as FMRP, to regulate target mRNA transport and local translation. The Arg87 variants may affect formation of these mRNA-regulatory protein complexes, or their function in regulating target mRNAs. 
with $\sim 102$ diverse membrane proteins, including protocadherins, G protein-coupled receptors (GPCRs), and ion channels (59). Importantly, a detailed structural analysis revealed that both the CYFIP and $A B I$ of the WRC formed an interaction surface toward those membrane proteins. Therefore, the CYFIP2 variants found in West syndrome may indirectly affect the interactions between the WRC and certain ion channels, possibly leading in turn to changes in both the ion channel properties and neuronal excitability (Fig. 1C). Furthermore, the FMRP-mediated regulation of expression of the proteins involved in modulating the synaptic E/I balance or the neuronal excitability may also play a role (60). Similarly to CYFIP1, CYFIP2 can form a complex with the FMRP and other RNA-binding proteins to regulate mRNA transport and local translation (40), whereas the Arg87 variants may affect this process (Fig. 1D). An additional comprehensive analysis of the expression, localization, interaction, and activity-dependent regulation of CYFIP2 using various in vitro and in vivo approaches will elucidate its unique functions, which can have implications for both the proper neuronal development and the pathophysiology of several brain disorders.

\section{ACKNOWLEDGEMENTS}

This work was supported by the National Research Foundation of Korea (NRF) grant funded by the Korea government Ministry of Science and ICT (NRF-2018R1C1B6001235, NRF-2018 R1A6A3A11040508), and by the Brain Research Program through the NRF funded by the Korea government Ministry of Science and ICT (NRF-2015M3C7A1028790, NRF-2018M3 C7A1024603).

\section{CONFLICTS OF INTEREST}

The authors have no conflicting interests.

\section{REFERENCES}

1. Marin O, Valiente M, Ge X and Tsai LH (2010) Guiding neuronal cell migrations. Cold Spring Harbor Persp Biol 2, a001834

2. Kevenaar JT and Hoogenraad CC (2015) The axonal cytoskeleton: from organization to function. Front $\mathrm{Mol}$ Neurosci 8, 44

3. Spence EF and Soderling SH (2015) Actin out: regulation of the synaptic cytoskeleton. J Biol Chem 290, 2861328622

4. Yan Z, Kim E, Datta D, Lewis DA and Soderling SH (2016) Synaptic actin dysregulation, a convergent mechanism of mental disorders? J Neurosci 36, 11411-11417

5. Choi S-Y and Han K (2015) Emerging role of synaptic actin-regulatory pathway in the pathophysiology of mood disorders. Animal Cells and Systems 19, 283-288

6. Penzes $\mathrm{P}$, Cahill ME, Jones KA, VanLeeuwen JE and Woolfrey KM (2011) Dendritic spine pathology in neuropsychiatric disorders. Nat Neurosci 14, 285-293
7. Takenawa T and Suetsugu S (2007) The WASP-WAVE protein network: connecting the membrane to the cytoskeleton. Nature reviews. Mol Cell Biol 8, 37-48

8. Chen Z, Borek D, Padrick SB et al (2010) Structure and control of the actin regulatory WAVE complex. Nature 468, 533-538

9. Mendoza MC (2013) Phosphoregulation of the WAVE regulatory complex and signal integration. Semin Cell Develop Biol 24, 272-279

10. Krause M and Gautreau A (2014) Steering cell migration: lamellipodium dynamics and the regulation of directional persistence. Nat Rev Mol Cell Biol 15, 577-590

11. Lee Y, Kim D, Ryu JR et al (2017) Phosphorylation of CYFIP2, a component of the WAVE-regulatory complex, regulates dendritic spine density and neurite outgrowth in cultured hippocampal neurons potentially by affecting the complex assembly. Neuroreport 28, 749-754

12. Schenck A, Bardoni B, Moro A, Bagni C and Mandel JL (2001) A highly conserved protein family interacting with the fragile $X$ mental retardation protein (FMRP) and displaying selective interactions with FMRP-related proteins FXR1P and FXR2P. Proc Natl Acad Sci U S A 98, 8844-8849

13. Bagni C, Tassone F, Neri G and Hagerman R (2012) Fragile $\mathrm{X}$ syndrome: causes, diagnosis, mechanisms, and therapeutics. J Clin Invest 122, 4314-4322

14. Napoli I, Mercaldo V, Boyl PP et al (2008) The fragile X syndrome protein represses activity-dependent translation through CYFIP1, a new 4E-BP. Cell 134, 1042-1054

15. De Rubeis S, Pasciuto E, Li KW et al (2013) CYFIP1 coordinates mRNA translation and cytoskeleton remodeling to ensure proper dendritic spine formation. Neuron 79, 1169-1182

16. Abekhoukh S and Bardoni B (2014) CYFIP family proteins between autism and intellectual disability: links with Fragile X syndrome. Front Cellular Neurosci 8, 81

17. Yoon KJ, Nguyen HN, Ursini G et al (2014) Modeling a genetic risk for schizophrenia in iPSCs and mice reveals neural stem cell deficits associated with adherens junctions and polarity. Cell Stem Cell 15, 79-91

18. Oguro-Ando A, Rosensweig C, Herman E et al (2015) Increased CYFIP1 dosage alters cellular and dendritic morphology and dysregulates mTOR. Mol Psychi 20, 1069-1078

19. Bozdagi O, Sakurai T, Dorr N, Pilorge M, Takahashi N and Buxbaum JD (2012) Haploinsufficiency of Cyfip1 produces fragile X-like phenotypes in mice. PloS one 7, e42422

20. Chung L, Wang X, Zhu L et al (2015) Parental origin impairment of synaptic functions and behaviors in cytoplasmic FMRP interacting protein 1 (Cyfip1) deficient mice. Brain Res 1629, 340-350

21. Kumar V, Kim K, Joseph C et al (2013) C57BL/6N mutation in cytoplasmic FMRP interacting protein 2 regulates cocaine response. Science 342, 1508-1512

22. Han K, Chen H, Gennarino VA, Richman R, Lu HC and Zoghbi HY (2015) Fragile X-like behaviors and abnormal cortical dendritic spines in cytoplasmic FMR1-interacting protein 2-mutant mice. Human Mol Gen 24, 1813-1823

23. Zhang $Y$, Kang $H$, Lee $Y$ et al (2019) Smaller body size, 
early postnatal lethality, and cortical extracellular matrix-related gene expression changes of Cyfip2-null embryonic mice. Front Mol Neurosci 11, 482

24. Nakashima $M$, Kato $M$, Aoto $K$ et al (2018) De Novo hotspot variants in CYFIP2 cause early-onset epileptic encephalopathy. Ann Neurol 83, 794-806

25. Peng J, Wang Y, He F et al (2018) Novel west syndrome candidate genes in a Chinese cohort. CNS Neurosci Ther 24, 1196-1206

26. Pathania $M$, Davenport EC, Muir J, Sheehan DF, Lopez-Domenech G and Kittler JT (2014) The autism and schizophrenia associated gene CYFIP1 is critical for the maintenance of dendritic complexity and the stabilization of mature spines. Translational Psychiatry 4, e374

27. Sala C and Segal M (2014) Dendritic spines: the locus of structural and functional plasticity. Physiological Reviews 94, 141-188

28. Collins MO, Husi H, Yu L et al (2006) Molecular characterization and comparison of the components and multiprotein complexes in the postsynaptic proteome. J Neurochem 97 Suppl 1, 16-23

29. Bayes A, van de Lagemaat LN, Collins MO et al (2011) Characterization of the proteome, diseases and evolution of the human postsynaptic density. Nat Neurosci 14, $19-21$

30. Li J, Zhang W, Yang H et al (2017) Spatiotemporal profile of postsynaptic interactomes integrates components of complex brain disorders. Nat Neurosci 20, 1150-1161

31. Han K, Holder JL Jr, Schaaf CP et al (2013) SHANK3 overexpression causes manic-like behaviour with unique pharmacogenetic properties. Nature 503, 72-77

32. Huttlin EL, Jedrychowski MP, Elias JE et al (2010) A tissue-specific atlas of mouse protein phosphorylation and expression. Cell 143, 1174-1189

33. Schenck A, Bardoni B, Langmann C, Harden N, Mandel JL and Giangrande A (2003) CYFIP/Sra-1 controls neuronal connectivity in drosophila and links the Rac1 GTPase pathway to the fragile $X$ protein. Neuron 38, 887-898

34. Pan L, Zhang YQ, Woodruff E and Broadie K (2004) The Drosophila fragile $X$ gene negatively regulates neuronal elaboration and synaptic differentiation. Curr Biol 14, 18 63-1870

35. Abekhoukh S, Sahin HB, Grossi M et al (2017) New insights into the regulatory function of CYFIP1 in the context of WAVE- and FMRP-containing complexes. Dis Mod Mech 10, 463-474

36. Zhao L, Wang D, Wang Q, Rodal AA and Zhang YQ (2013) Drosophila cyfip regulates synaptic development and endocytosis by suppressing filamentous actin assembly. PLoS Genetics 9, e1003450

37. Trowe $\mathrm{T}$, Klostermann S, Baier $\mathrm{H}$ et al (1996) Mutations disrupting the ordering and topographic mapping of axons in the retinotectal projection of the zebrafish, Danio rerio. Development 123, 439-450

38. Baier $\mathrm{H}$, Klostermann $\mathrm{S}$, Trowe $\mathrm{T}$, Karlstrom RO, Nusslein-Volhard C and Bonhoeffer F (1996) Genetic dissection of the retinotectal projection. Development 123, 415-425

39. Pittman AJ, Gaynes JA and Chien CB (2010) nev (cyfip2) is required for retinal lamination and axon guidance in the zebrafish retinotectal system. Develop Biol 344, 784-794

40. Cioni JM, Wong HH, Bressan D, Kodama L, Harris WA and Holt CE (2018) Axon-axon interactions regulate topographic optic tract sorting via CYFIP2-dependent WAVE complex function. Neuron 97, 1078-1093 e1076

41. Marsden KC, Jain RA, Wolman MA et al (2018) A Cyfip2-Dependent Excitatory Interneuron Pathway Establishes the Innate Startle Threshold. Cell Rep 23, 878-887

42. Kirkpatrick SL, Goldberg LR, Yazdani N et al (2017) Cytoplasmic FMR1-Interacting protein 2 is a major genetic factor underlying binge eating. Biological Psychiatry 81, 757-769

43. Keane TM, Goodstadt L, Danecek P et al (2011) Mouse genomic variation and its effect on phenotypes and gene regulation. Nature 477, 289-294

44. Chen B, Chou HT, Brautigam CA et al (2017) Rac1 GTPase activates the WAVE regulatory complex through two distinct binding sites. eLife 6, e29795

45. Darnell JC, Van Driesche SJ, Zhang C et al (2011) FMRP stalls ribosomal translocation on mRNAs linked to synaptic function and autism. Cell 146, 247-261

46. Lee JH, Kim HJ, Yoon JM et al (2016) Interstitial deletion of 5 q33.3q35.1 in a boy with severe mental retardation. Korean J Pediat 59, S19-S24

47. Spranger S, Rommel B, Jauch A, Bodammer R, Mehl B and Bullerdiek J (2000) Interstitial deletion of $5 q 33.3 q 35.1$ in a girl with mild mental retardation. Am J Med Genet 93, 107-109

48. Focking M, Lopez LM, English JA et al (2015) Proteomic and genomic evidence implicates the postsynaptic density in schizophrenia. Mol Psych 20, 424-432

49. Tiwari SS, Mizuno K, Ghosh A et al (2016) Alzheimerrelated decrease in CYFIP2 links amyloid production to tau hyperphosphorylation and memory loss. Brain : J Neurol 139, 2751-2765

50. Gursoy S and Ercal D (2016) Diagnostic Approach to Genetic Causes of Early-Onset Epileptic Encephalopathy. J Child Neurol 31, 523-532

51. McTague A, Howell KB, Cross JH, Kurian MA and Scheffer IE (2016) The genetic landscape of the epileptic encephalopathies of infancy and childhood. Lancet Neurol 15, 304-316

52. Bonaccorso CM, Spatuzza M, Di Marco B et al (2015) Fragile $X$ mental retardation protein (FMRP) interacting proteins exhibit different expression patterns during development. Int J Devel Neurosci 42, 15-23

53. Zhang Y, Chen K, Sloan SA et al (2014) An RNA-sequencing transcriptome and splicing database of glia, neurons, and vascular cells of the cerebral cortex. J Neurosci 34, 11929-11947

54. Bassell GJ and Warren ST (2008) Fragile X syndrome: loss of local mRNA regulation alters synaptic development and function. Neuron 60, 201-214

55. Cajigas IJ, Tushev G, Will TJ, tom Dieck S, Fuerst N and Schuman EM (2012) The local transcriptome in the synaptic neuropil revealed by deep sequencing and high-resolution imaging. Neuron 74, 453-466

56. Nelson SB and Valakh V (2015) Excitatory/Inhibitory balance and circuit homeostasis in autism spectrum 
disorders. Neuron 87, 684-698

57. Lee Y, Zhang Y, Kim S and Han K (2018) Excitatory and inhibitory synaptic dysfunction in mania: an emerging hypothesis from animal model studies. Exp Mol Med 50, 12

58. Hsiao K, Harony-Nicolas H, Buxbaum JD, Bozdagi-Gunal O and Benson DL (2016) Cyfip1 Regulates Presynaptic
Activity during Development. J Neurosci 36, 1564-1576

59. Chen B, Brinkmann K, Chen Z et al (2014) The WAVE regulatory complex links diverse receptors to the actin cytoskeleton. Cell 156, 195-207

60. Contractor A, Klyachko VA and Portera-Cailliau C (2015) altered neuronal and circuit excitability in fragile $X$ syndrome. Neuron 87, 699-715 